\title{
Association of ultrasonographic features with histologic findings in 7l dogs with protein-losing nephropathy (2008-2018)
}

Lucy Kopecny, BVSc (Hons) ${ }^{1 *}$; Carrie A. Palm, DVM, MAS ${ }^{1}$; Kelsey Brust, DVM ${ }^{2}$; Michelle A. Giuffrida, VMD, MSCE ${ }^{2}$; Larry D. Cowgill, DVM, PhD ${ }^{1}$; Eric G. Johnson, DVM ${ }^{2}$

'Department of Medicine and Epidemiology, School of Veterinary Medicine, University of California-Davis, Davis, CA

${ }^{2}$ Department of Surgical and Radiological Sciences, School of Veterinary Medicine, University of California-Davis, Davis, CA

*Corresponding author: Dr. Kopecny (Ikopecny@sashvets.com)

\section{OBJECTIVE}

To determine whether ultrasonographic features in dogs with protein-losing nephropathy (PLN) were associated with renal biopsy findings and compare corticomedullary ratios between dogs with PLN versus non-renal disease.

\section{ANIMALS}

7I dogs with PLN and 33 dogs without renal disease.

\section{PROCEDURES}

Medical records and archived ultrasonographic images for dogs with PLN that underwent renal biopsy between 2008 and 2018 were reviewed. Corticomedullary ratios were measured.

\section{RESULTS}

In dogs with PLN, median serum creatinine and BUN concentrations and urine-protein-to-creatinine-ratio prior to renal biopsy were $3.4 \mathrm{mg} / \mathrm{dL}$ (interquartile range [IQR], 1.2 to $5.3 \mathrm{mg} / \mathrm{dL}$ ), $80 \mathrm{mg} / \mathrm{dL}$ (IQR, 28 to $105 \mathrm{mg} / \mathrm{dL}$ ), and II.4 (IQR, 6.4 to I8.3), respectively. Histologic abnormalities within the tubulointerstitial space were associated with cortical echogenicity. Gastric wall thickness $>5 \mathrm{~mm}$ was associated with a histologic diagnosis of acute glomerular disease. Dogs with immune complexmediated glomerular disease were more likely to have abnormal gastric mural architecture. Other ultrasonographic features of the kidneys, liver, and stomach and the presence of ascites did not help to differentiate immune complex-mediated from non-immune complex-mediated glomerular disease, acute from chronic disease, or amyloid from non-amyloid disease or distinguish whether tubulointerstitial disease was present or absent. Median left corticomedullary ratio for 66 dogs with PLN (I.2) was significantly higher than that for the 33 dogs without renal disease (I.0).

\section{CLINICAL RELEVANCE}

Ultrasonographic features were poorly associated with specific pathological disorders in dogs with PLN. In this study, the corticomedullary ratio was higher in dogs with PLN, indicating the presence of cortical thickening, but the clinical relevance is unknown.

$\mathrm{W}$ ith the application of light, transmission electron, and immunofluorescence microscopy for evaluation of renal biopsy specimens from dogs, it has been possible to distinguish various pathological patterns of glomerular disease and differentiate immune complexmediated from non-immune complex-mediated glomerular diseases. ${ }^{1}$ Immune complex-mediated glomerular disease has been identified in $48.1 \%$ of dogs in a North American study ${ }^{2}$ undergoing renal biopsy because of suspected glomerular disease and $50.6 \%$ of dogs in a European study. ${ }^{3}$ Corresponding ultrasonographic features of these diseases and whether ultrasonographic features can be used to differentiate specific pathological patterns are not known.
Ultrasonographic imaging is routinely used to screen for structural changes of the kidneys. Several studies $^{4-9}$ have investigated the ultrasonographic features associated with various nephropathies in dogs. In people, studies examining correlations between histopathologic and ultrasonographic findings have yielded mixed results..$^{10}$ Researchers have evaluated the corticomedullary ratio in healthy cats ${ }^{11,12}$ and in people with and without renal disease ${ }^{13}$; however, to our knowledge, this ratio has not been investigated in dogs without evidence of renal disease or in dogs with biopsy-confirmed renal diseases such as protein-losing nephropathy (PLN). Subjective ultrasonographic assessment of renal cortical thickness at our 
institution has indicated that dogs with PLN might have a thicker renal cortex.

The objectives of the retrospective study reported here were to describe ultrasonographic features in dogs with PLN and to determine whether ultrasonographic features in dogs with PLN were associated with renal biopsy findings. We also aimed to objectively compare corticomedullary ratio between dogs with PLN and dogs with ultrasonographically normal kidneys and normal renal functional parameters. We hypothesized that ultrasonographic features would be associated with histopathologic changes in dogs with PLN and that renal cortical thickness would be greater in dogs with PLN than in dogs without renal disease.

\section{Materials and Methods}

\section{Case selection criteria and medical records review}

Medical records of the University of CaliforniaDavis William R. Pritchard Veterinary Medical Teaching Hospital were searched to identify all dogs that underwent renal biopsy for evaluation of PLN between January 1, 2008, and December 31, 2018. Dogs were eligible for inclusion in the study if they had undergone abdominal ultrasonography $\leq 30$ days prior to undergoing renal biopsy and if renal biopsy specimens had been evaluated by means of light microscopy and, when indicated, immunofluorescence and transmission electron microscopy.

Medical records of dogs included in the study were reviewed, and information on breed, sex, neuter status, age, body weight, and blood pressure at the time of renal biopsy was recorded for each dog. Clinicopathologic data extracted from the medical records consisted of the most recent $\mathrm{CBC}$, serum biochemistry panel, routine urinalysis, and urine aerobic bacterial culture results and urine protein-tocreatinine concentration ratio (UPCR) obtained prior to renal biopsy. When available, plasma antithrombin concentration was also recorded. Duration of disease was defined by the date on which proteinuria was first identified.

The medical records database was also searched to identify dogs with presenting complaints unrelated to the urogenital system that underwent a complete abdominal ultrasonographic examination between January 1, 2008, and December 31, 2018. This population comprised a convenience sample of dogs presenting for non-urogenital disease. Dogs representing a range of ages and body weights were selected to ensure a comprehensive population. Dogs were eligible for inclusion if renal function, as determined by laboratory evaluation, was normal. Specifically, dogs were required to have a serum creatinine concentration $<1.5 \mathrm{mg} / \mathrm{dL}$, a BUN concentration $<33 \mathrm{mg} / \mathrm{dL}$, and a urine specific gravity $\geq 1.030$ with no evidence of proteinuria on a routine urinalysis performed $\leq$ 30 days prior to the ultrasonographic examination. These dogs did not undergo renal biopsy.

\section{Renal biopsy}

For each dog with PLN, the method used to collect renal biopsy specimens was recorded. Renal biopsy specimens were submitted to the International Veterinary Renal Pathology Service at The Ohio State University and the William R. Pritchard Veterinary Medical Teaching Hospital. On the basis of the renal biopsy report, glomerular disease was characterized as immune complex-mediated or non-immune complex-mediated glomerular disease and as acute or chronic disease. Chronicity was determined by the presence of glomerulosclerosis, fibrous and fibrocellular crescents, interstitial fibrosis, and tubular atrophy. ${ }^{1,14}$ The presence or absence of concurrent tubulointerstitial disease and amyloid deposits was recorded. Renal lesions were categorized by the nephropathologist as membranoproliferative glomerulonephritis, membranous glomerulonephropathy, mesangioproliferative glomerulonephritis, mixed immune complex-mediated glomerulonephritis (including mixed membranoproliferative glomerulonephritis and mixed membranous glomerulonephropathy), focal segmental glomerulosclerosis, amyloidosis, and end-stage renal disease, according to established pathological criteria. ${ }^{1,15}$ End-stage renal disease was characterized histologically by severe interstitial fibrosis, tubular atrophy, mononuclear cell inflammation, and global glomerulosclerosis or glomerular obsolescence. ${ }^{16}$

\section{Ultrasonographic examination}

For dogs with PLN, archived ultrasonographic images obtained $\leq 30$ days before renal biopsy were reviewed by 2 board-certified radiologists (EGJ and KB) who achieved consensus on image interpretation. Ultrasonography had been performed with either of 2 machines (iE33 [Phillips Ultrasound Inc] or HDI 5000 [Phillips Ultrasound Inc]) with an 8- to 5-mHz curved-array transducer. Ultrasonographic images were reviewed at a single DICOM workstation with standard viewing software (eFilm workstation; Merge Healthcare). Ultrasonographic features of the kidneys that were evaluated included corticomedullary distinction, cortical and medullary echogenicity, capsular margin contour, presence of cortical infarcts, cortical cystic lesions, cortical mineralization, medullary rim sign, and subcapsular fluid.

Corticomedullary distinction was graded subjectively as normal, increased, or decreased. Cortical and medullary echogenicity were graded as normal, increased, or decreased by comparison with echogenicities of adjacent normal-appearing organs, if included in archived images, or by subjective assessment of the renal parenchyma itself.,17,18 Capsular margin contour was graded as smooth or irregular. Infarcts were recorded as present if focal wedgeshaped or striated hyperechoic or hypoechoic regions were seen in the renal cortex with capsular margin contour irregularities. Cortical cysts were defined as round, anechoic, thin-walled structures 
confined to the cortex. Cortical mineralization was defined as small hyperechoic foci in the renal cortical tissues. A medullary rim sign was recorded if a thin, hyperechoic line was present in the medulla, parallel to the corticomedullary junction. Subcapsular fluid was noted if curvilinearly distributed anechoic fluid was evident along the peripheral margin of the kidney cortex just deep to the renal capsule.

Corticomedullary ratio was determined on archived images of the left kidney taken in a sagittal plane of the kidney (Figure I). The left kidney was selected for analysis because higher-quality sagittal images were more consistently available than for the right kidney. Maximum renal medullary thickness was measured with a calibrated measuring tool over the renal crest and was measured from the junction of the renal medullary crest with the renal pelvis (demarcated by a thin single margin or parallel hyperechoic linear margins) to the outer medulla at the corticomedullary junction. Cortical thickness was measured with the ruler tool from the corticomedullary junction to the renal capsular margin. The corticomedullary ratio was calculated by dividing cortical thickness by medullary thickness. Overly oblique parasagittal images were excluded from calculations. Images were deemed oblique and not used if there was asymmetry to the renal crest or a lack of distinction of the junction of the renal medulla with the renal pelvis.

The presence of retroperitoneal or peritoneal fluid, the presence of hepatomegaly, relative hepatic echogenicity, gastric wall thickness, subjective gastric mucosal echogenicity, and the presence or absence of normal gastric mural architecture were recorded when possible. Hepatomegaly was defined as a liver with rounded borders that projected beyond the gastric margin. Hepatic echogenicity was subjectively evaluated by comparison with adjacent normal-appearing organs and portal vein conspicuity. A gastric wall thickness $\geq 5 \mathrm{~mm}$ was considered abnormal. ${ }^{19}$

For dogs without renal disease or proteinuria, renal architecture, echogenicity, and echotexture were deemed normal by 2 board-certified veterinary radiologists (EGJ and KB) when evaluated subjectively and in comparison to adjacent normal-appearing organs and previously described imaging findings. ${ }^{7,17,18}$ The corticomedullary ratio was measured in these dogs as described for dogs with PLN.

\section{Statistical analysis}

Descriptive statistics were calculated. Continuous variables were evaluated for a normal distribution by means of a combined test for skewness and kurtosis. Continuous variables are reported as mean and SD if data were normally distributed and as median and interquartile (25th to 75 th percentile) range (IQR) if data were not normally distributed. Categorical variables are reported as counts and percentages.
The corticomedullary ratio was compared between dogs with PLN and dogs without renal disease by means of the Wilcoxon rank sum test.

Fisher exact or Wilcoxon rank sum tests were used to determine whether specific defined ultrasonographic features were significantly associated with the presence of immune complex-mediated versus non-immune complex-mediated glomerular disease, the presence of acute versus chronic glomerular disease, the presence versus absence of concurrent tu-
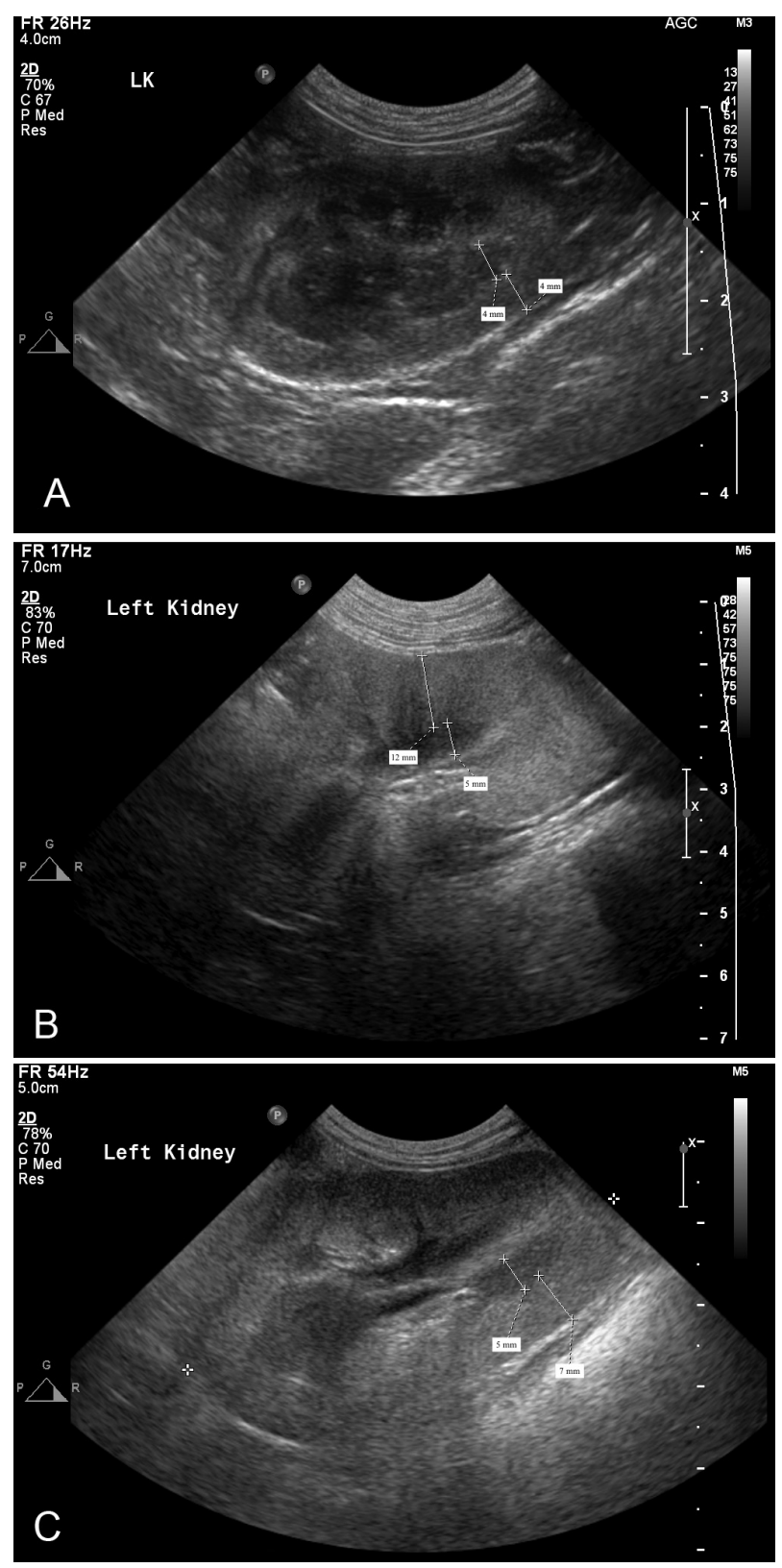

Figure I-Archived ultrasound images showing measurement of corticomedullary ratio in a healthy dog (A), a dog with amyloidosis (B), and a dog with focal segmental glomerulosclerosis (C). The maximum renal medullary thickness was measured from the junction of the renal medullary crest with the renal pelvis (demarcated by a thin single margin or paralleling hyperechoic linear margins) to the outer medulla at the corticomedullary junction. The cortex was measured from the corticomedullary junction to the renal capsular margin. 
bulointerstitial disease, and the presence of amyloid versus non-amyloid glomerular disease.

Statistical analyses were performed with standard software (Stata Statistical Software release 16; StataCorp LLC). All tests were 2 -sided, with values of $P<0.05$ considered significant.

\section{Results}

\section{Dogs without renal disease}

Thirty-three dogs without renal disease met the inclusion criteria. Presenting complaints included neurologic disease $(n=28)$, clinical trial enrollment (4), and immune-mediated polyarthritis (1). Mean \pm SD age was $4.7 \pm 3.1$ years. Median weight was 26.7 $\mathrm{kg}$ (IQR, 13.8 to $40.0 \mathrm{~kg}$ ). Mean serum creatinine concentration was $1.0 \pm 0.2 \mathrm{mg} / \mathrm{dL}$ (reference range, 0.8 to $1.5 \mathrm{mg} / \mathrm{dL}$ ), mean BUN concentration was $16 \pm 5$ $\mathrm{mg} / \mathrm{dL}$ (reference range, 11 to $33 \mathrm{mg} / \mathrm{dL}$ ), mean albumin concentration was $3.7 \pm 0.3 \mathrm{~g} / \mathrm{dL}$ (reference range, 3.4 to $4.3 \mathrm{~g} / \mathrm{dL}$ ), and median globulin concentration was $2.5 \mathrm{~g} / \mathrm{dL}$ (IQR, 2.3 to 2.7 ; reference range, 1.7 to $3.1 \mathrm{~g} / \mathrm{dL}$ ). Mean urine specific gravity was 1.041 \pm 0.008 . Mean left corticomedullary ratio was $1.0 \pm$ 0.2 (median, 1.0; IQR, 0.9 to 1.1).

\section{Dogs with PLN}

Seventy-one dogs with PLN met the inclusion criteria. An additional 13 dogs were identified as having undergone renal biopsy but were excluded because abdominal ultrasonography was not performed $\leq 30$ days before renal biopsy $(n=7)$, renal biopsy was performed for evaluation of non-proteinuric renal disease (4), or transmission electron microscopy was not performed, preventing accurate categorization into listed morphological diagnosis categories (2).

Mean \pm SD age of the dogs with PLN was $7.1 \pm$ 2.9 years, and mean body weight was $21.5 \pm 12.8 \mathrm{~kg}$. There were 34 (47.9\%) spayed females, 29 (40.8\%) castrated males, $6(8.5 \%)$ sexually intact males, and $2(2.8 \%)$ sexually intact females. Fifteen of the 71 (21.1\%) dogs were of mixed breeding. The most common purebred dogs were Golden Retrievers $(\mathrm{n}=4[5.6 \%])$, Labrador Retrievers (4 [5.6\%]), Miniature Schnauzers $(3[4.2 \%])$, Shar Pei $(3[4.2 \%])$, Standard Poodles (3 [4.2\%]), and Yorkshire Terriers (3 [4.2\%]). Median time from the diagnosis of proteinuria to renal biopsy was 28 days (IQR, 13 to 78 days).

Clinicopathologic data-Median urine protein-tocreatinine concentration ratio before renal biopsy was 11.4 (IQR, 6.4 to 18.3; reference range, < 0.2; Table I). Antithrombin concentration was measured before renal biopsy in 49 of the 71 dogs; median concentration was $54 \%$ (IQR, $42.5 \%$ to $75 \%$; reference range, $80 \%$ to $120 \%$ ). Testing for anti-Borrelia burgdorferi antibodies was performed in 55 dogs; 6 (10.9\%) of these dogs were positive for antibodies.
Median serum creatinine concentration $(3.1 \mathrm{mg} /$ dL [IQR, 1.1 to $4.1 \mathrm{mg} / \mathrm{dL}$ ] vs $3.5 \mathrm{mg} / \mathrm{dL}$ [IQR, 1.2 to $5.4 \mathrm{mg} / \mathrm{dL}$ ], respectively; $P=0.5$ ), BUN concentration $(96 \mathrm{mg} / \mathrm{dL}$ [IQR, 33 to $101 \mathrm{mg} / \mathrm{dL}$ ] vs $67 \mathrm{mg} / \mathrm{dL}$ [IQR, 28 to $105 \mathrm{mg} / \mathrm{dL}$ ], respectively; $P=0.39$ ), and serum albumin concentration ( $2.0 \mathrm{~g} / \mathrm{dL}$ [IQR, 1.8 to $2.5 \mathrm{~g} /$ dL] vs $2.5 \mathrm{~g} / \mathrm{dL}$ [IQR, 2.0 to $3.0 \mathrm{~g} / \mathrm{dL}$ ], respectively; $P$ $=0.05$ ) did not differ significantly between dogs with acute versus chronic glomerular disease.

Histologic findings-Renal biopsy was performed by means of ultrasound-guided needle biopsy in 57 of the $71(80.3 \%)$ dogs, exploratory laparotomy in 9 $(12.7 \%)$, and laparoscopic-guided needle biopsy in 5 (7.0\%). Specimens were collected by needle biopsy for 7 of the 9 dogs that underwent exploratory laparotomy, by punch biopsy for 1 , and by wedge biopsy for 1 .

Morphological diagnoses included membranoproliferative glomerulonephritis $(\mathrm{n}=19$ [26.8\%]), focal segmental glomerulosclerosis (19 [26.8\%]), amyloidosis (13 [18.3\%]), membranous glomerulonephropathy (11 [15.5\%]), mesangioproliferative glomerulonephritis (4 [5.6\%]), mixed immune complex-mediated glomerulonephritis (2 [2.8\%]), and end-stage renal disease $(2[2.8 \%])$. In the remaining dog, the disease could not be categorized as a distinct morphological diagnosis; this dog had non-immune complex-mediated glomerulopathy with amyloid deposits in approximately $25 \%$ of glomeruli in addition to moderate chronic-active tubulointerstitial nephritis.

Overall, 37 of 71 (52.1\%) dogs had immune complex-mediated glomerular disease and 34 (47.9\%) had non-immune complex-mediated glomerular disease, 14 (20.0\%) dogs had acute glomerular disease and 56 (80.0\%) had chronic glomerular disease, 50 (70.4\%) dogs had concurrent tubulointerstitial disease and 21 (29.6\%) did not have concurrent tubulointerstitial disease, and 14 (19.7\%) dogs had amyloid deposits and 57 $(80.3 \%)$ had non-amyloid glomerular disease.

Ultrasonographic findings-Renal corticomedullary distinction was normal in 13 of the $70(18.6 \%)$ dogs with PLN, increased in 12 (17.1\%), and decreased

Table I-Results of clinicopathologic testing performed $\leq \mathbf{3 0}$ days prior to renal biopsy in 7l dogs with protein-losing nephropathy (PLN).

\begin{tabular}{|c|c|c|}
\hline Variable & Median (IQR) & Reference range \\
\hline Hct (\%) & 37.1 (32.4-42.9) & $40-55$ \\
\hline Neutrophils (cells/ $\mu \mathrm{L}$ ) & $8,575(5,586-11,595)$ & $3,000-10,500$ \\
\hline $\begin{array}{l}\text { Platelets } \\
\left(\times 10^{3} \text { platelets } / \mu \mathrm{L}\right)\end{array}$ & $271.5(212-427.8)$ & $150-400$ \\
\hline Creatinine $(\mathrm{mg} / \mathrm{dL})$ & $3.4(1.2-5.3)$ & $0.8-1.5$ \\
\hline BUN (mg/dL) & $80(28-105)$ & $11-33$ \\
\hline Albumin (g/dL) & $2.4(1.9-2.9)$ & $3.4-4.3$ \\
\hline Globulin (g/dL) & $2.4(2.1-2.7)$ & I.7-3.| \\
\hline Cholesterol (mg/dL) & $306(257-398)$ & $139-353$ \\
\hline Urine specific gravity & $1.016(1.013-1.021)$ & $>1.030$ \\
\hline
\end{tabular}

$\mathrm{IQR}=$ Interquartile (25th to 75 th percentile) range. 
in $45(64.3 \%)$. Renal cortical echogenicity was normal in 6 of the 70 (8.6\%) dogs and increased in 64 (91.4\%). Renal medullary echogenicity could not be assessed in 2 dogs but was normal in 26 of 69 (37.7\%) dogs, increased in $42(60.9 \%)$, and decreased in 1 (1.4\%). Retroperitoneal fluid was identified in 8 of the 70 (11.4\%) dogs, and peritoneal fluid was identified in $28(40.0 \%)$. Of the $8 \mathrm{dogs}$ with retroperitoneal fluid, $3(37.5 \%)$ were classified as having acute glomerular disease. Cortical cysts were present in 15 of the 71 (21.1\%) dogs, cortical mineralization was present in 10 (14.1\%), cortical infarcts were present in $1(1.4 \%)$, and a medullary rim sign was detected in 19 (26.8\%). Renal capsular margin contour was irregular in 2 (2.9\%) of the 68 dogs in which it could be assessed and smooth in the remaining 66 dogs. Subcapsular fluid was identified in 2 of the 71 (2.8\%) dogs.

Corticomedullary distinction (normal vs increased vs decreased), cortical echogenicity (normal vs increased), medullary echogenicity (normal vs increased vs decreased), presence of retroperitoneal fluid (yes vs no), and presence of peritoneal fluid (yes vs no) did not differ significantly between dogs with immune complex-mediated versus non-immune complex-mediated glomerular disease, between dogs with acute versus chronic glomerular disease, between dogs with versus without concurrent tubulointerstitial disease, or between dogs with amyloid versus non-amyloid glomerular disease (Tables 2-5), except that cortical echogenicity was significantly $(P$ $=0.008$ ) more likely to be increased in dogs with concurrent tubulointerstitial disease than in dogs without concurrent tubulointerstitial disease. Of dogs with histopathologic evidence of tubulointerstitial disease for which results of cortical echogenicity evaluation were available, 48 of 49 (98.0\%) had increased cortical echogenicity, and 16 of 21 (76.2\%) dogs without histopathologic evidence of tubulointerstitial disease had increased cortical echogenicity.

The liver was visible on ultrasonographic images of 68 of the dogs with PLN. Hepatomegaly was present in 23 of 66 (33.8\%) dogs (liver size could not be assessed in 2 dogs), with liver echogenicity considered normal in 32 of 68 (47.1\%) dogs, increased in 22 (32.4\%), and decreased in 14 (20.6\%). The percentage of dogs with hepatomegaly did not differ significantly $(P=0.09)$ between dogs with non-amyloid glomerular disease (16/54 [29.6\%]) and dogs with amyloid glomerular disease (7/12 [58.3\%]). Similarly, hepatic echogenicity did not differ significantly $(P=0.23)$ between dogs with amyloid glomerular disease (normal, 9/14 [64.3\%]; increased, 2/14 [14.3\%], and decreased, $3 / 14$ [21.4\%]) and dogs with non-amyloid glomerular disease (normal, 23/54 [42.6\%]; increased, 20/54 [37.0\%]; and decreased, 11/54 [20.4\%]).

Gastric wall thickness could be assessed in 58 dogs and was $>5 \mathrm{~mm}$ in 23 (39.7\%). Gastric mural architecture could be assessed in 50 dogs and was abnormal in 26 (52.0\%). Gastric mucosal echogenicity could be assessed in 54 dogs and was increased in 13 (24.1\%), decreased in 14 (25.9\%), and normal in 27 (50.0\%). Gastric mucosal echogenicity did not differ significantly between dogs with immune complexmediated versus non-immune complex-mediated glomerular disease, between dogs with acute versus chronic glomerular disease, between dogs with versus without concurrent tubulointerstitial disease, or between dogs with amyloid versus non-amyloid disease (Tables 2-5). Dogs with immune complexmediated glomerular disease were significantly $(P=$

Table 2-Ultrasonographic findings for 7I dogs with PLN determined, on the basis of histologic examination of renal biopsy specimens, to have immune complex-mediated $(n=37)$ or non-immune complex-mediated (34) glomerular disease.

\begin{tabular}{|c|c|c|c|}
\hline Variable & $\begin{array}{c}\text { Non-immune } \\
\text { complex-mediated }\end{array}$ & $\begin{array}{c}\text { Immune } \\
\text { complex-mediated }\end{array}$ & $P$ value \\
\hline Corticomedullary distinction & & & 0.94 \\
\hline Normal & $6(18.2)$ & 7 (18.9) & \\
\hline Increased & $5(15.2)$ & $7(18.9)$ & \\
\hline Decreased & $22(66.7)$ & $23(62.2)$ & \\
\hline Cortical echogenicity & & & 0.68 \\
\hline Normal & $2(6.1)$ & $4(10.8)$ & \\
\hline Increased & 31 (93.9) & $33(89.2)$ & \\
\hline Medullary echogenicity & & & 0.80 \\
\hline Normal & $12(37.5)$ & $14(37.8)$ & \\
\hline Increased & $19(59.4)$ & $23(62.2)$ & \\
\hline Decreased & I (3.I) & $0(0.0)$ & \\
\hline Retroperitoneal fluid & $5(15.2)$ & $3(8.1)$ & 0.46 \\
\hline Peritoneal fluid & $10(30.3)$ & $18(48.7)$ & 0.15 \\
\hline Gastric wall $>5 \mathrm{~mm}^{\mathrm{a}}$ & $8(29.6)$ & $15(48.4)$ & 0.18 \\
\hline Gastric wall echogenicity ${ }^{b}$ & & & 0.10 \\
\hline Normal & $16(66.7)$ & II (36.7) & \\
\hline Increased & $4(16.7)$ & $9(30.0)$ & \\
\hline Decreased & $4(16.7)$ & $10(33.3)$ & \\
\hline Abnormal gastric mural architecture & $6(30.0)$ & $20(66.7)$ & 0.02 \\
\hline
\end{tabular}


Table 3-Ultrasonographic findings for 7I dogs with PLN determined, on the basis of histologic examination of renal biopsy specimens, to have acute $(n=14)$ or chronic $(57)$ glomerular disease.

\begin{tabular}{|c|c|c|c|}
\hline Variable & Acute & Chronic & $P$ value \\
\hline Corticomedullary distinction & & & 0.10 \\
\hline Normal & I (7.I) & $12(21.4)$ & \\
\hline Increased & $5(35.7)$ & $7(12.5)$ & \\
\hline Decreased & $8(57.1)$ & $37(66.1)$ & \\
\hline Cortical echogenicity & & & $>0.99$ \\
\hline Normal & I (7.I) & $5(8.9)$ & \\
\hline Increased & $13(92.9)$ & 51 (9I.I) & \\
\hline Medullary echogenicity & & & 0.49 \\
\hline Normal & $7(50.0)$ & $19(34.6)$ & \\
\hline Increased & $7(50.0)$ & $35(63.6)$ & \\
\hline Decreased & $0(0.0)$ & $\mathrm{I}(\mathrm{I} .8)$ & \\
\hline Retroperitoneal fluid & $3(21.4)$ & $5(8.9)$ & 0.19 \\
\hline Peritoneal fluid & $9(64.3)$ & $19(33.9)$ & 0.07 \\
\hline Gastric wall > $5 \mathrm{~mm}^{\mathrm{a}}$ & $9(81.8)$ & $14(29.8)$ & 0.004 \\
\hline Gastric wall echogenicity ${ }^{b}$ & & & 0.05 \\
\hline Normal & $2(18.2)$ & $25(58.1)$ & \\
\hline Increased & $4(36.4)$ & $9(20.9)$ & \\
\hline Decreased & $5(45.5)$ & $9(20.9)$ & \\
\hline Abnormal gastric mural architecture ${ }^{c}$ & $8(72.7)$ & $18(46.2)$ & 0.18 \\
\hline
\end{tabular}

See Table 2 for key.

Table 4-Ultrasonographic findings for 7I dogs with PLN that had $(n=50)$ or did not have $(2 \mathrm{I})$ concurrent tubulointerstitial disease.

\begin{tabular}{|c|c|c|c|}
\hline Variable & $\begin{array}{c}\text { No tubulointerstitial } \\
\text { disease }\end{array}$ & $\begin{array}{c}\text { Tubulointerstitial } \\
\text { disease }\end{array}$ & $P$ value \\
\hline Corticomedullary distinction & & & 0.31 \\
\hline Normal & $6(28.6)$ & $7(14.3)$ & \\
\hline Increased & $4(19.1)$ & $8(16.3)$ & \\
\hline Decreased & II (52.4) & $34(69.4)$ & \\
\hline Cortical echogenicity & & & 0.008 \\
\hline Normal & $5(23.8)$ & I (2.0) & \\
\hline Increased & $16(76.2)$ & $48(98.0)$ & \\
\hline Medullary echogenicity & & & 0.12 \\
\hline Normal & $10(47.6)$ & $16(33.3)$ & \\
\hline Increased & $10(47.6)$ & $32(66.7)$ & \\
\hline Decreased & I (4.8) & $0(0.0)$ & \\
\hline Retroperitoneal fluid & $3(15.0)$ & $5(10.0)$ & 0.68 \\
\hline Peritoneal fluid & $9(45.0)$ & $19(38.0)$ & 0.60 \\
\hline Gastric wall $>5 \mathrm{~mm}^{\mathrm{a}}$ & $5(3 \mid .3)$ & $18(42.9)$ & 0.55 \\
\hline Gastric wall echogenicity ${ }^{b}$ & & & 0.53 \\
\hline Normal & $9(60.0)$ & $18(46.2)$ & \\
\hline Increased & $2(13.3)$ & II (28.2) & \\
\hline Decreased & $4(26.7)$ & $10(25.6)$ & \\
\hline Abnormal gastric mural architecture ${ }^{c}$ & $7(50.0)$ & $19(52.8)$ & $>0.99$ \\
\hline
\end{tabular}

See Table 2 for key.

0.02) more likely to have abnormal gastric mural architecture (20/30 [66.7\%]) than were dogs with nonimmune complex-mediated glomerular disease $(6 / 20$ [30.0\%]). Increased gastric wall thickness (ie, gastric wall thickness $>5 \mathrm{~mm})$ was significantly $(P=0.004)$ more common in dogs with acute glomerular disease (9/11 [81.8\%]) than in dogs with chronic glomerular disease (14/47 [29.8\%]).

Left corticomedullary ratio could be measured in 66 of the dogs with PLN. Median ratio for dogs with PLN (1.2; IQR, 1.0 to 1.4$)$ was significantly $(P<0.001)$ higher than the median ratio for the 33 dogs without renal disease (1.0; IQR, 0.9 to 1.1$)$. Median left corticomedullary ratio was not significantly different between dogs with immune complex-mediated glomerular disease (1.2; IQR, 1.0 to 1.4$)$ versus dogs with non-immune complex-mediated glomerular disease (1.1; IQR, 1.0 to $1.4 ; P=0.97)$, between dogs with acute glomerular disease (1.1; IQR, 0.9 to 1.3$)$ versus chronic glomerular disease (1.2; IQR. 1.0 to $1.4 ; P=$ 0.18 ), between dogs with concurrent tubulointerstitial disease (1.3; IQR, 1.0 to 1.5$)$ versus dogs without concurrent tubulointerstitial disease (1.1; IQR, 1.0 to $1.2 ; P=0.11)$, or between dogs with amyloid glomerular disease (1.1; IQR, 1.0 to 1.4$)$ versus non-amyloid glomerular disease (1.2; IQR, 1.0 to $1.4 ; P=0.91)$. 
Table 5-Ultrasonographic findings for 7I dogs with PLN determined, on the basis of histologic examination of renal biopsy specimens, to have non-amyloid $(n=56)$ or amyloid $(14)$ glomerular disease.

\begin{tabular}{lccc} 
Variable & Non-amyloid & Amyloid & P value \\
\hline Corticomedullary distinction & & & $>0.99$ \\
$\quad$ Normal & $10(17.9)$ & $3(21.4)$ & \\
Increased & $10(17.9)$ & $2(14.3)$ & \\
Decreased & $36(64.3)$ & $9(64.3)$ & \\
Cortical echogenicity & & & \\
$\quad$ Normal & $5(8.9)$ & $1(7.1)$ & 0.99 \\
Increased & $51(91.1)$ & $13(92.9)$ & \\
Medullary echogenicity & & & \\
Normal & $21(38.2)$ & $5(35.7)$ & \\
Increased & $34(61.8)$ & $8(57.1)$ & 0.66 \\
Decreased & $0(0.0)$ & $1(7.1)$ & 0.77 \\
Retroperitoneal fluid & $6(10.7)$ & $2(14.3)$ & 0.50 \\
Peritoneal fluid & $23(41.1)$ & $5(35.7)$ & 0.71 \\
Gastric wall >5 mma & $20(42.6)$ & $3(27.3)$ & \\
Gastric wall echogenicity & & & \\
Normal & $22(48.9)$ & $5(55.6)$ & $>0.99$ \\
Increased & $12(26.7)$ & $1(11.1)$ & 0.09 \\
Decreased & $11(24.4)$ & $3(33.3)$ & 0.23 \\
Abnormal gastric mural architecturec & $22(52.4)$ & $7(50.0)$ & \\
Hepatomegaly & $16(29.6)$ & $9(58.3)$ & \\
Hepatic echogenicity & $23(42.6)$ & $9(64.3)$ & \\
Normal & $20(37.0)$ & $3(21.4)$ & \\
Increased & $11(20.4)$ & & \\
Decreased & & \\
\hline
\end{tabular}

See Table 2 for key.

\section{Discussion}

In the present study, abdominal ultrasonographic features evaluated in dogs with PLN were poorly associated with specific histopathologic patterns of glomerular disease. This finding emphasizes the importance of renal biopsy as the gold standard for diagnosis of glomerular disease in $\operatorname{dogs}^{1}$; however, abdominal ultrasonography remains a valuable diagnostic aid in evaluating these dogs because it can help identify complications secondary to PLN and infectious, inflammatory, and neoplastic diseases that could lead to glomerulopathies. ${ }^{20}$ Additionally, abdominal ultrasonography allows evaluation of the kidneys for other diseases contributing to decreased renal function, such as pyelonephritis, ureteral obstruction, and chronic kidney disease. ${ }^{7,9,21}$

The sonographically calculated corticomedullary ratio was higher in dogs with PLN than in dogs with normal renal function and normal ultrasonographic architecture in our study. This finding supports the presence of cortical thickening in dogs with PLN; however, corticomedullary ratio cannot be used to further define the underlying disease in dogs with PLN, and although the difference between groups was statistically significant, it was small. Cortical thickening might occur as a result of hypercellularity of the glomerulus in some cases, with or without infiltration of cells into the tubulointerstitium. ${ }^{22} \mathrm{Re}-$ nal enlargement secondary to these changes is recognized in people, although cortical thickening is not specifically reported. ${ }^{22}$ Alternatively, the perceived thickening of the renal cortex could be artifactual secondary to renal abnormalities at the corticomedullary interface creating the sonographic appearance of a thickened cortex. Thus, correlation with results of full-thickness renal biopsy specimens is a future direction that should be explored. Measurements from the left kidney were used to determine corticomedullary ratio in dogs in the present study, because high-quality archived sagittal images of the left kidney were more often available than those of the right kidney. This was likely a result of the relative ease of ultrasonographic imaging of the left kidney, compared with the right. Because glomerular disease is generally diffuse and bilateral ${ }^{16}$ the use of images of only the left kidney was considered unlikely to affect our results. However, we were not able to test for differences in corticomedullary ratio between kidneys in dogs with PLN in the present study.

There was no difference in the corticomedullary ratio between dogs with chronic versus acute glomerular disease in our study, though this is recognized in humans. ${ }^{23}$ However, it is likely dogs with more severe chronic disease were less likely to undergo renal biopsy owing to technical considerations of renal biopsy collection in dogs and the poor perceived prognosis in dogs with severe chronic disease regardless of renal biopsy findings. Inclusion of dogs with more severe chronic glomerulopathies might have resulted in a difference in corticomedullary ratio between dogs with chronic disease and dogs with acute disease.

In the present study, dogs with concurrent tubulointerstitial disease were more likely to have increased renal cortical echogenicity than were dogs 
without concurrent tubulointerstitial disease. Overall, however, 91.4\% (64/70) of the dogs with PLN in the present study had increased cortical echogenicity. Therefore, it is unlikely that increased cortical echogenicity will be clinically useful in identifying tubulointerstitial disease. In dogs, ultrasonographic cortical echogenicity is a poor discriminatory test between normal and chronically diseased kidneys. ${ }^{5,6}$ In people with a range of renal diseases, including glomerular disease, interstitial inflammation and tubular atrophy are important determinants of renal cortical echogenicity, whereas interstitial fibrosis is not correlated with echogenicity. ${ }^{24}$ Therefore, by focusing on specific tubulointerstitial abnormalities such as the presence of interstitial inflammation, tubular atrophy, and fibrosis, it might be possible to detect additional associations between specific histopathologic findings and cortical echogenicity. This was not possible in the present study owing to the small number of dogs with tubulointerstitial changes on renal biopsy.

Gastric wall thickening is reported in dogs with azotemia and associated with specific histopathologic changes, including edema and mucosal or submucosal mineralization. ${ }^{4,25}$ In the present study, dogs with acute glomerular disease were more likely to have gastric wall thickening (ie, gastric wall thickness $>5 \mathrm{~mm}$ ) than were dogs with chronic glomerular disease, which might be clinically useful. Surprisingly, this finding was present despite a lack of significant differences in serum creatinine, BUN, or albumin concentrations between these groups. It appears disease severity, based on degree of azotemia and hypoalbuminemia, does not account for gastric wall thickening in this cohort and other comorbidities (eg, acute vomiting) might be contributing to gastric wall changes. Additionally, it is unknown what compensatory mechanisms occur in chronic renal disease that might mitigate the degree of gastric wall thickening. This is in contrast to a study ${ }^{25}$ showing that more severe azotemia was associated with histopathologic gastric wall changes in dogs. Differences in gastric wall features between dogs with acute versus chronic renal disease, either sonographic or histopathologic, have not been described previously to the authors' knowledge.

There were no ultrasonographic features that enabled differentiation of dogs with amyloidosis from dogs with other glomerular diseases in the present study. This included ultrasonographic features of the liver and stomach, even though extrarenal amyloid deposits are reported in $73 \%$ of Shar Pei dogs and $29 \%$ of non-Shar Pei dogs. ${ }^{26}$ Although the ability to differentiate amyloidosis from other glomerular diseases on the basis of ultrasonographic features would be beneficial in avoiding renal biopsy for a disease with no specific treatment, the findings in the present study suggested that ultrasonographic changes to the kidneys and extrarenal organs are non-specific in dogs with amyloidosis. There were only small numbers of dogs with amyloidosis in this study (13 dogs, representing $18.3 \%$ of dogs with PLN in the study population), and not all extra-renal abdominal organs that can be affected by amyloid deposition (including the pancreas and spleen $)^{26}$ were evaluated.

Immune complex-mediated glomerular disease was identified in 37 of the 71 (52.1\%) dogs in the present study, which was similar to percentages reported in other studies. ${ }^{2,3}$ Membranoproliferative glomerulonephritis and focal segmental glomerulosclerosis were the most often diagnosed glomerulopathies, with 19 of the 70 (26.8\%) dogs having each of these glomerulopathies.

The present study had several limitations. The study population of dogs with PLN was based on availability, and it is possible that additional associations existed but were not identified as significant because some of the subgroups used for comparison were somewhat small. Interobserver variability was limited by having archived ultrasonographic images reviewed by 2 board-certified veterinary radiologists. However, there was variation in the archived ultrasonographic images that were available for review, resulting in missing observations for some dogs. In addition, differences in the sagittal plane of archived images of the kidney could have contributed to some variation in the calculated corticomedullary ratio. Prospective ultrasonographic evaluation of dogs with PLN would allow collection of additional measurements such as kidney-to-aorta ratio and kidney-tolumbar vertebral body ratio for estimation of kidney size in dogs with PLN. ${ }^{27,28}$

In summary, ultrasonographic features were poorly associated with specific histopathologic diagnoses in dogs with PLN in the present study. As such, renal biopsy will continue to be critical for accurate diagnosis of this condition in dogs. The sonographically determined corticomedullary ratio was slightly higher in dogs with PLN than in dogs with normal renal function, suggesting cortical thickening may be present in dogs with PLN; however, further research with full-thickness renal biopsy specimens is needed to corroborate this finding. Additionally, gastric wall thickening was more likely to be associated with acute renal disease as opposed to chronic renal disease.

\section{Acknowledgments}

No third-party funding or support was received in connection with this study or the writing or publication of the manuscript. The authors declare that there were no conflicts of interest.

\section{References}

1. Cianciolo RE, Brown CA, Mohr FC, et al. Pathologic evaluation of canine renal biopsies: methods for identifying features that differentiate immune-mediated glomerulonephritides from other categories of glomerular diseases. $J$ Vet Intern Med. 2013;27(suppl 1):S10-S18. doi:10.1111/jvim.12226

2. Schneider SM, Cianciolo RE, Nabity MB, Clubb FJ Jr, Brown CA, Lees GE. Prevalence of immune-complex glomerulonephritides in dogs biopsied for suspected glomerular disease: 501 cases (2007-2012). J Vet Intern Med. 2013;27(suppl 1):S67-S75. doi:10.1111/jvim.12247 
3. Aresu L, Martini V, Benali SL, et al. European Veterinary Renal Pathology Service: a survey over a 7-year period (20082015). J Vet Intern Med. 2017;31(5):1459-1468. doi:10.1111/ jvim. 14796

4. Grooters AM, Miyabayashi T, Biller DS, Merryman J. Sonographic appearance of uremic gastropathy in 4 dogs. Vet Radiol Ultrasound. 1994;35(1):35-40. doi:10.1111/j.1740-8261.1994. tb00175.x

5. Zotti A, Banzato T, Gelain ME, Centelleghe C, Vaccaro C, Aresu L. Correlation of renal histopathology with renal echogenicity in dogs and cats: an ex-vivo quantitative study. BMC Vet Res. 2015;11:99. doi:10.1186/s12917-015-0415-8

6. Banzato T, Bonsembiante F, Aresu L, Zotti A. Relationship of diagnostic accuracy of renal cortical echogenicity with renal histopathology in dogs and cats, a quantitative study. BMC Vet Res. 2017;13(1):24. doi:10.1186/s12917-016-0941-z

7. Bragato N, Borges NC, Fioravanti MCS. B-mode and Doppler ultrasound of chronic kidney disease in dogs and cats. Vet Res Commun. 2017;41(4):307-315. doi:10.1007/s11259-0179694-9

8. Mantis P, Lamb CR. Most dogs with medullary rim sign on ultrasonography have no demonstrable renal dysfunction. Vet Radiol Ultrasound. 2000;41(2):164-166. doi:10.1111/j.1740-8261.2000.tb01471.x

9. D'Anjou MA, Bédard A, Dunn ME. Clinical significance of renal pelvic dilatation on ultrasound in dogs and cats. Vet Radiol Ultrasound. 2011;52(1):88-94. doi:10.1111/j.17408261.2010.01729.x

10. Hricak H, Cruz C, Romanski R, et al. Renal parenchymal disease: sonographic-histologic correlation. Radiology. 1982;144(1):141-147. doi:10.1148/radiology.144.1.7089245

11. Debruyn K, Paepe D, Daminet S, et al. Comparison of renal ultrasonographic measurements between healthy cats of three cat breeds: Ragdoll, British Shorthair and Sphynx.J Feline Med Surg. 2013;15(6):478-482. doi:10.1177/1098612X12471057

12. Debruyn K, Paepe D, Daminet S, et al. Renal dimensions at ultrasonography in healthy Ragdoll cats with normal kidney morphology: correlation with age, gender and bodyweight. J Feline Med Surg. 2013;15(12):1046-1051. doi:10.1177/1098612X13487361

13. Khademi M. Angiographic measurement of renal compart ments. Corticomedullary ratio in normal, diseased states and sickle cell anemia. Radiology. 1974;113(1):51-58. doi:10.1148/113.1.51

14. Chang A, Gibson IW, Cohen AH, Weening JW, Jennette JC, Fogo AB. A position paper on standardizing the nonneoplastic kidney biopsy report. Clin J Am Soc Nepbrol. 2012;7(8):1365-1368. doi:10.2215/CJN.02300312

15. Cianciolo RE, Mohr FC, Aresu L, et al. World Small Animal Veterinary Association Renal Pathology Initiative: classification of glomerular diseases in dogs. Vet Patbol. 2016;53(1):113135. doi:10.1177/0300985815579996
16. Breshears MA, Confer AW. The urinary system. In: Zachary JF, ed. Pathologic Basis of Veterinary Disease. 6th ed. Elsevier; 2017:617-681.

17. Nyland TG, Widmer WR, Mattoon JS. Urinary tract. In: Mattoon JS, Nyland TG, eds. Small Animal Diagnostic Ultrasound. 3rd ed. W.B. Saunders; 2015:557-607.

18. Ivancić M, Mai W. Qualitative and quantitative comparison of renal vs. hepatic ultrasonographic intensity in healthy dogs. Vet Radiol Ultrasound. 2008;49(4):368-373. doi:10.1111/ j.1740-8261.2008.00383.x

19. Penninck DG, Nyland TG, Fisher PE, Kerr LY. Ultrasonography of the normal canine gastrointestinal tract. Vet $\mathrm{Ra}$ diol. 1989;30(6):272-276. doi:10.1111/j.1740-8261.1989. tb01799.x

20. IRIS Canine GN Study Group Diagnosis Subgroup, Littman MP, Daminet S, Grauer GF, Lees GE, van Dongen AM. Consensus recommendations for the diagnostic investigation of dogs with suspected glomerular disease. J Vet Intern Med. 2013;27(suppl 1):S19-S26. doi:10.1111/jvim.12223

21. Walter PA, Feeney DA, Johnston GR, Oleary TP. Ultrasonographic evaluation of renal parenchymal diseases in dogs: 32 cases (1981-1986). J Am Vet Med Assoc. 1987;191(8):9991007 .

22. Page JE, Morgan SH, Eastwood JB, et al. Ultrasound findings in renal parenchymal disease: comparison with histological appearances. Clin Radiol. 1994;49(12):867-870. doi:10.1016/ s0009-9260(05)82877-6

23. Roger SD, Beale AM, Cattell WR, Webb JA. What is the value of measuring renal parenchymal thickness before renal biopsy. Clin Radiol. 1994;49(1):45-49. doi:10.1016/s00099260(05)82913-7

24. Moghazi S, Jones E, Schroepple J, et al. Correlation of renal histopathology with sonographic findings. Kidney Int. 2005;67(4):1515-1520. doi:10.1111/j.1523-1755.2005.00230.x

25. Peters RM, Goldstein RE, Erb HN, Njaa BL. Histopathologic features of canine uremic gastropathy: a retrospective study. J Vet Intern Med. 2005;19(3):315-320. doi:10.1892/08916640(2005)19[315:hfocug]2.0.co;2

26. Segev G, Cowgill LD, Jessen S, Berkowitz A, Mohr CF, Aroch I. Renal amyloidosis in dogs: a retrospective study of 91 cases with comparison of the disease between Shar-Pei and non-Shar-Pei dogs. J Vet Intern Med. 2012;26(2):259-268. doi:10.1111/j.1939-1676.2011.00878.x

27. Barella G, Lodi M, Sabbadin LA, Faverzani S. A new method for ultrasonographic measurement of kidney size in healthy dogs. J Ultrasound. 2012;15(3):186-191. doi:10.1016/j. jus.2012.06.004

28. Mareschal A, d'Anjou MA, Moreau M, Alexander K, Beauregard G. Ultrasonographic measurement of kidney-toaorta ratio as a method of estimating renal size in dogs. Vet Radiol Ultrasound. 2007;48(5):434-438. doi:10.1111/j.17408261.2007.00274.x 